\title{
Population Vector Coding by the Giant Interneurons of the Cockroach
}

\author{
Rafael Levi and Jeffrey M. Camhi \\ Department of Cell and Animal Biology, Life Sciences Institute, Hebrew University, Jerusalem, Israel 91904
}

We tested two alternative models of integration among the cockroach giant interneurons (Gls) for determining the directions of wind-evoked escape turns. One model, called steering wheel, pits contralateral Gls against one another; the other, called population vector model, involves a vector computation among the Gls. In testing each model theoretically, the population vector was found to account far better for the actual behavior. Both models could account for the results of previous behavioral-physiological experiments in which spikes had been added to the right GI3 together with wind stimuli from the right side. The two models revealed a critical behavioral-physiological experimental test that we then performed; namely, when delivering wind from the right side, adding spikes experimen-

Animals often select and perform a given form of a particular behavior, from among several possible alternative forms: for instance, moving in one versus another direction. In this paper, we analyze the mechanisms by which a group of giant interneurons (GIs) determine the direction of the escape behavior in the cockroach.

In some directional behaviors of other animals, mutual inhibition among interneurons is essential in selecting direction. This inhibition effectively cancels, or vetoes, the activity of all but one interneuron (Wine and Krasne, 1972; Eaton et al., 1991) or one coherent set of interneurons (Salzman and Newsome, 1994), giving rise to a "winner-take-all" situation. The "winning" interneuron or set evokes a turn in a direction determined by its unique output connections. In the cockroach escape system, the direction of the behavior has been shown not to result from a winner-take-all mechanism (Levi and Camhi, 2000).

In other behaviors, rather than one cell vetoing the action of another, the interneurons collaborate to determine direction. For instance, in the abdominal posture system of the crayfish, electrically stimulating any of several interneurons results in a graded alteration of abdominal posture. Some interneurons elevate the abdomen and others depress it. The moment-to-moment abdominal posture is determined by the partial ongoing effects of the many such interneurons of both of these groups, which feed onto tonic elevator or depressor motor neurons (Evoy and Kennedy,

Received Oct. 18, 1999; revised Jan. 13, 2000; accepted Feb. 11, 2000.

This work was supported by United States of America-Israel Binational Science Foundation Grant 93-00021/3. We thank Prof. Apostolos Georgopoulos and Erez Ezrahi for valuable advice and assistance.

Correspondence should be addressed to Jeffrey M. Camhi, Department of Cell and Animal Biology, Hebrew University, Givat-Ram, Jerusalem, 91904 Israel. E-mail: jeff@vms.huji.ac.il.

Dr. Levi's present address: Department of Biology, University of California, San Diego, La Jolla, CA 92093-0357.

Copyright (C) 2000 Society for Neuroscience $\quad 0270-6474 / 00 / 203822-08 \$ 15.00 / 0$ tally to the right GI2 should increase turn size according to the steering wheel model but should decrease turn size according to the population vector model. The latter result was obtained. The population vector, but not the steering wheel, model also could account for previous behavioral-physiological experiments in which spikes were added experimentally to a Gl contralateral to the wind stimuli. The results support the population vector model as accounting for direction determination among the cockroach Gls.

Key words: escape behavior; electrical stimulation; neural control; population coding; population vector; giant interneurons; interneuron; cockroach

1967; Kennedy et al., 1967). In the present paper, we test a model of cockroach escape that we call steering wheel, which closely resembles this crayfish system.

An alternative form of cellular collaboration is the population vector code (Sparks et al., 1976; Georgopoulos et al., 1986; Groh et al., 1997). In the control of a monkey's arm movement, for instance (Georgopoulos et al., 1986), activity of a given neuron of the motor cortex promotes a given direction of movement. The more spikes this cell gives, the more effective it is in promoting its preferred movement direction. A given cell thus "attracts" the movement of the arm, from any other direction, toward its own preferred direction. This attraction from all other directions would not occur in the crayfish example cited above (or in the steering wheel model we develop here); rather, more spikes in, say, a crayfish abdominal depressor interneuron, could only cause more abdominal depression and never elevation.

In this paper, we show that, theoretically, the steering wheel model can account only partly for the cockroach's directional decision, whereas the population vector model accounts for it well. Moreover, we show that the cockroach's system of giant interneurons attracts the turn form all directions. These results point toward the population vector, and not the steering wheel, model as accounting for the direction of cockroach escape.

\section{MATERIALS AND METHODS}

We used adult male cockroaches, Periplaneta americana, in all experiments. We raised the cockroaches at $26^{\circ} \mathrm{C}$, on a $12 \mathrm{hr}$ light/dark cycle, in 50 gallon screened cages. The cockroaches were fed rat chow and water ad libitum.

Behavioral methods. The cockroach exhibits the normal leg movements of escape when tethered on a slick surface (Camhi and Levy, 1988; Nye and Ritzmann, 1992). Such tethering permits controlled sensory stimulation, as well as both intracellular recording and stimulation of GIs during the evoked escape behavior (Liebenthal et al., 1994; Kolton and Camhi, 1995; Levi and Camhi, 2000).

The behavioral testing system has been described previously 
(Liebenthal et al., 1994; Kolton and Camhi, 1995; Levi and Camhi, 2000). Briefly, we tethered the cockroach on glass coated with mineral oil. We delivered controlled wind puff stimuli from different azimuthal directions. The peak wind intensity was $1.2 \mathrm{~m} / \mathrm{sec}$, as measured with a hot wire anemometer (Flow Corp., Watertown, MA) at the location of the cerci. We delivered wind puffs from different angles on the animal's right side. Throughout this paper, we designate the anterior end of the animal as $0^{\circ}$, its posterior end as $180^{\circ}$, and due right as $90^{\circ}$.

For measuring the cockroach's turning direction, we recorded windevoked changes of the coxa-femur (CF) joint for each leg. This joint movement has been shown to vary systematically with turn direction (Nye and Ritzmann, 1992; Levi and Camhi, 2000). To record these joint movements, we used a high-speed video ( 250 frames/sec; NAC, Tokyo, Japan). We then analyzed the CF joint movements frame by frame on a personal computer, using a video analysis program (MTV; Data Crunch, San Clemente, CA). As described previously (Levi and Camhi, 2000), we measured the joint angle, one frame before an escape response began, and again three frames $(12 \mathrm{msec})$ later. Subtracting the first angle from the second yielded the joint movement. Combining the joint movements of all six legs gave a measure of left-turning tendency. For this, we added the joint movements of all six legs, each multiplied by a coefficient derived by multiple linear regression between the six joint movements and the applied wind angle. Left-turning tendency ranges from 0 (no turn) to 1 (largest mean turn size, for winds from $30^{\circ}$ right). Armed with this measure of the relative strength of a given left turn away from a right wind stimulus, it was possible for us to interpret the cockroach's behavioral responses to our experimental alteration of spike trains in particular GIs.

Physiological methods. We used standard methods for intracellular recording from the GIs, using glass microelectrodes with an impedance range of 20-40 M $\Omega$ (Liebenthal et al., 1994). The electrodes were back filled with $6 \%$ carboxy fluorescein and filled with $3 \mathrm{M} \mathrm{KCl}$. For intracellular stimulation of GIs, we used the discontinuous current-clamp mode of an Axoclamp 2B amplifier (Axon Instruments, Foster City, CA). This method enabled us to deliver $1 \mathrm{msec}$ pulses of more than $100 \mathrm{nA}$ and still record the evoked action potentials with the same electrode. We stored all physiological data on videotapes using a Neurocorder (Neuro Data, New York, NY). We then analyzed the stored data with the help of a personal computer program (Computerscope; RC Electronics, Santa Barbara, CA). For calculation of instantaneous frequency, we used the inverse of the interspike intervals, taken from the computer program at $460 \mathrm{sec}$ resolution.

At the end of each experiment, we injected the cell by passing hyperpolarizing current of up to $100 \mathrm{nA}$ for 10-20 min and identified the cell in a whole mount using a fluorescence microscope (Standard; Zeiss, Oberkochen, Germany). (Some of this high current may have leaked from the axon, because the cockroach's running movements caused some decrease in the quality of the electrode penetration.) In some experiments, a high intensity CCD camera (C2400; Hamamtsu, Tokyo, Japan) aided in visualization.

We recorded extracellular activity of the whole nerve cord with a pair of silver hook electrodes positioned underneath the nerve cord. The extracellular activity recorded was amplified with an AC amplifier (Grass P15; Grass Instruments, Quincy, MA) and was used as an indication of both the healthy condition of the nerve cord and the success of the intracellular stimulation.

In some experiments, we killed a portion of a GI by means of photoablation (Miller and Selverston, 1979; Libersat et al., 1989). This entailed penetrating the axon in the A5-A6 connective with a microelectrode, filling the axon with $6 \%$ carboxy fluorescein by means of $100 \mathrm{nA}$ hyperpolarizing DC current for $10 \mathrm{~min}$, and irradiating the nerve cord with a spot of $8 \mathrm{~mm}$ diameter from a $200 \mathrm{~W}$ fiber optic apparatus (Olympus 3001; Olympus Optical, Tokyo, Japan) through a blue excitation filter (Libersat and Mizrahi, 1996). The spot was directed on the posterior region of the A5-A6 connective and the A6 ganglion. In experiments in which we wanted to follow this procedure with electrical stimulation of the GI axons more proximally, we filled the axon as just described, and then we removed the microelectrode and repenetrated the GI in the A4-A5 connective. We then irradiated as before. As a result, only the posterior region of the filled axon was killed, leaving the newly impaled region of the axon and its entire more anterior length intact. From the newly penetrated region, it was possible to record the stages of the local axonal death produced by the posterior photoablation (Libersat et al., 1989), verifying that we had indeed repenetrated the same axon. Overall, this treatment eliminated the sensory input to the GI but enabled us to stimulate the axon and record the evoked action potentials by using the discontinuous current-clamp mode of the Axoclamp amplifier.

Mathematical procedures. The least-square error optimization of the steering wheel coefficients was performed using the program Matlab on UNIX. The population vector calculation was performed by a custom program on a personal computer. All data from test results reported in the text are given as mean \pm SEM

\section{RESULTS}

\section{Two models of giant interneuron collaboration to determine turn direction}

A group of six GIs are centrally involved in deciding the direction of the cockroach's escape turn: left and right GIs 1, 2, and 3 (Comer, 1985; Liebenthal et al., 1994; Levi and Camhi, 2000). The relative numbers or frequencies of action potentials in these different GIs constitute a critical parameter in deciding the turn direction. Fine temporal pattering within the spike trains of the GIs, although present, appears to play little or no role (Liebenthal et al., 1994; Levi and Camhi, 2000).

These GIs are part of a wind-activated escape circuit. A wind gust produced by an approaching predator evokes in the cockroach a turn away from the stimulus, followed by rapid running (Camhi and Tom, 1978). The wind sensory cells, each directionsensitive, are located on the two posterior appendages called cerci, and their axons converge on the GIs. Owing to the patterns of convergence, each GI has a unique directional response to wind (Kolton and Camhi, 1995) (Fig. 1A). The GI axons project from the last abdominal ganglion to the three thoracic ganglia in which they activate further groups of interneurons, and ultimately the leg motor neurons.

One model that could, in principle, account for the determination of turn direction by GIs is called here steering wheel and is shown in Figure $1 B$ (top panel). (In the sketches of this and the following population vector model, we include for simplicity only the more directional GIs: left and right GI2 and GI3. Below, however, we test each model both with and without GI 1.) By this mechanism, each GI acts as though to turn a steering wheel in a given direction, either clockwise (thus contributing to a right turn) or counterclockwise (contributing to a left turn). The more spikes a given GI gives in response to wind, the stronger its effect. Thus, with the wind coming from the right (Fig. $1 A$ ), the counterclockwise arrows dominate (Fig. $1 B$ ), resulting in a left turn (Fig. $1 C$ ). In the simplest form of this mechanism, each spike in any GI would produce a turn of equivalent strength. As a slight elaboration of the mechanism that improves the model, different GIs are assigned different weightings of the effectiveness of each of their spikes. Note, for instance, that in Figure $1 B$, the circular arrow of right GI3 is longer than that of right GI2, although the wind in this model, from $90^{\circ}$ right, evokes more spikes in right GI2 than in right GI3 (Fig. $1 A$ ).

According to this steering wheel model, the final turn direction is calculated as the summation of all these partial pushes on the wheel: some clockwise and others counterclockwise, as seen in Figure $1 B$, bottom panel. If the wind were to come from an angle different from $90^{\circ}$ right, say closer to the right front, right GI3 would give more spikes than right GI2. Owing to the greater weighting of the spikes of GI3 than GI2, the cockroach would thus turn more sharply to the left. The steering wheel model is similar to models that have been presented before to account for the cockroach's turn direction (Dowd and Comer, 1988; Camhi, 1988) and shares with them the idea of a summation of spikes from the two sides pushing in opposite directions. This has been 
A Input

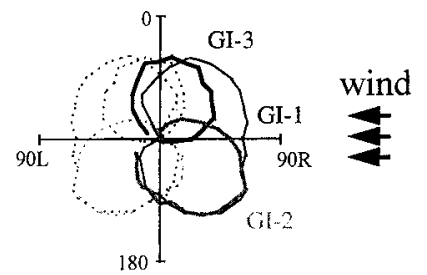

B Calculation

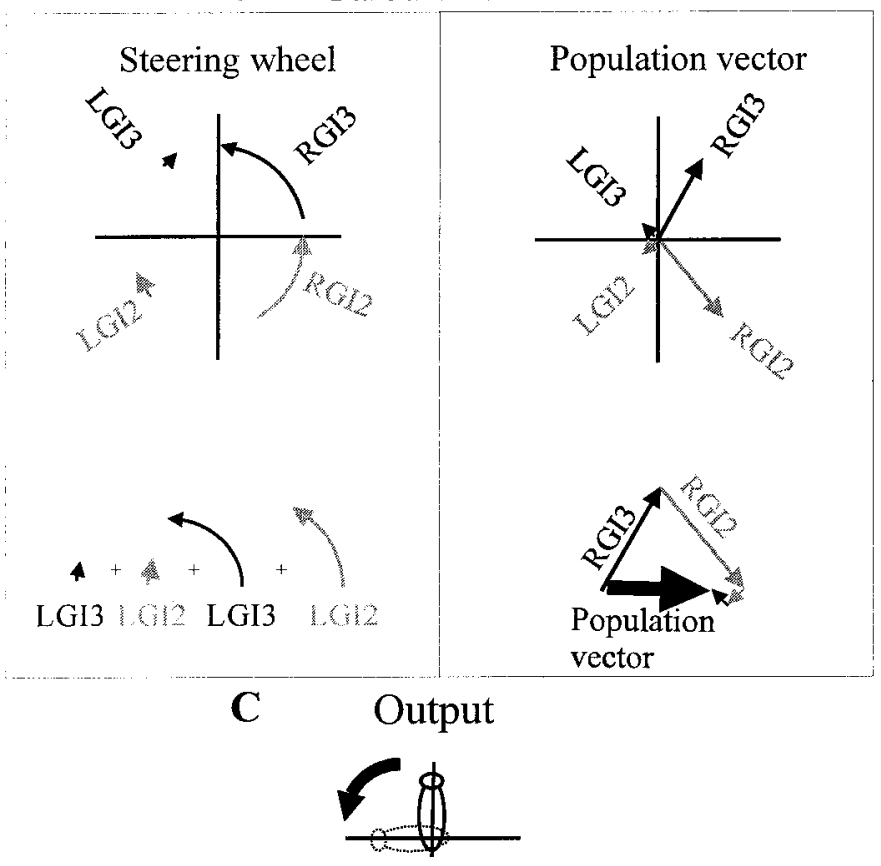

Figure 1. Steering wheel and population vector models, shown schematically. $A$, Input to each model: polar plots of the receptive fields of the GIs in response to wind from various angles about the cockroach in the horizontal plane. 0, Wind from the front; 180, wind from the rear; $90 R$, $90 \mathrm{~L}$, wind orthogonally from the right and left, respectively. Solid lines, Right GIs 1, 2, and 3; dotted lines, their left homologs. Plots show numbers of spikes during the first $45 \mathrm{msec}$ of the response to wind. Full length of each axis equals 10 spikes. The wind direction shown by the arrows (from $90^{\circ}$ right) is the angle used in $B$ and $C$ (modified from Kolton and Camhi, 1995). B, Steering wheel, The length of a given arrow reflects the number of spikes evoked in the given GI in response to the $90^{\circ}$ right wind stimulus. Arrow length also reflects differential weightings given to each GI (a GI3 spike being more effective than a GI2 spike). Arrows point in the directions of motor effect produced by each GI. The summation shown below would lead to a large left turn. Population vector, The direction of each arrow indicates the preferred wind direction of a given GI. The length of each arrow shows the number of spikes it gives in response to the $90^{\circ}$ right wind. The model calculates wind direction by vector summation (bottom panel). Turn direction is opposite the calculated wind direction. $C$, Turn direction produced by either the steering wheel or the population vector model.

the main conceptual framework to date for understanding the higher organization of this cockroach system.

A population vector model of the cockroach GIs is illustrated in Figure $1 C$. The preferred direction of wind stimulus for a given GI is shown by the arrow of that GI on the Cartesian coordinates. The length of each arrow corresponds to the number of spikes that GI gives in response to the wind stimulus from $90^{\circ}$ right (Fig. $1 A)$. A vector summation (Fig. $1 C$, bottom panel) gives the population vector, which corresponds to the direction from which the cockroach's nervous system, according to this model, would calculate the wind to have arrived. To execute the escape from this wind then, the cockroach would simply reverse the direction of the arrow and thus would turn left (Fig. 1C).

\section{Simulations of the steering wheel and population vector mechanisms}

Before performing physiological experiments to determine whether the cockroach GIs might use a steering wheel or a population vector mechanism, we tested each model theoretically to determine whether, and how well, each can account for the observed directional behavior. These models rely on the known receptive fields of each GI (Kolton and Camhi, 1995) (Fig. 1A), which were obtained in our laboratory, using wind stimuli with identical peak strength as in the present experiments, $1.2 \mathrm{~m} / \mathrm{sec}$. From these receptive field data, we obtained, for any given direction of wind stimulus, the number of spikes, on average, given by each left and right GI 1,2, and 3.

We first simulated the escape behavior based on the steering wheel model. For this, we began by using a linear summation of the numbers of spikes in each GI for right wind directions, between $0^{\circ}$ and $180^{\circ}$. We used the following equation to calculate the estimated turn: Turn size $=a(g i 1)+b(g i 2)+\mathrm{c}(g i 3)$, where $a, b$, and $c$ are the coefficients for left or right GI1, GI2, and GI3 respectively (contralaterally homologous GIs have the same coefficient), and gi1, gi2, and gi3, respectively, are the differences in the numbers of spikes between homologous GI pairs, for each wind angle (for instance, gil is the numbers of spikes in the right GI1 minus that in the left GI1).

To determine the coefficients $a, b$, and $c$, we used a least-square mean error procedure that minimized the difference between a theoretically "perfect" turn $\left(180^{\circ}\right.$ away from the wind source) and the linear sum. (In reality, cockroaches rarely achieve these perfect turns away from the wind source. However, introducing into the model more realistic turn size would merely involve a change of scale and thus would not disturb the theoretical analysis.) We restricted all coefficients to positive integers. The resulting coefficients gave the optimal linear transformation from the GI responses to the turn size. Based on this procedure, we obtained the following coefficients for the equation: $a=6.9, b=$ 7.2, and $c=28$.

Because the coefficient of GI3 is the largest, according to this optimized model each spike of the right GI3, which responds primarily to wind from the front right, would contribute more strongly to a left turn than would a spike in either right GI1 or GI2. This weighting of right GI3 is reflected in Figure $1 B$ (left panel, long circular arrow) for this GI.

Figure $2 A$ (filled diamonds) shows the resulting turn size as a function of wind direction, based on the above equation. The fit is reasonably close to the prediction (dashed line) for angles from $\sim 50^{\circ}$ to $180^{\circ}$, although it fails precipitously for lower wind angles (i.e., wind from near the front). The total root mean square (RMS) is 50, which we compare below with that of the population vector code model. The poor discrimination at frontal wind angles is caused by the small sum that results from the above equation for frontal winds in which opposite GIs nearly cancel out the effects of each other. (By this algorithm, small sums produce small turns.) Thus, at just those angles at which the cockroach actually makes the largest turns (close to $0^{\circ}$ ), this model predicts small turns. This suggests then that, if the steering wheel model does apply to this system, additional factors must be incorporated to correct this error in the frontal region. Given the reasonable fit 


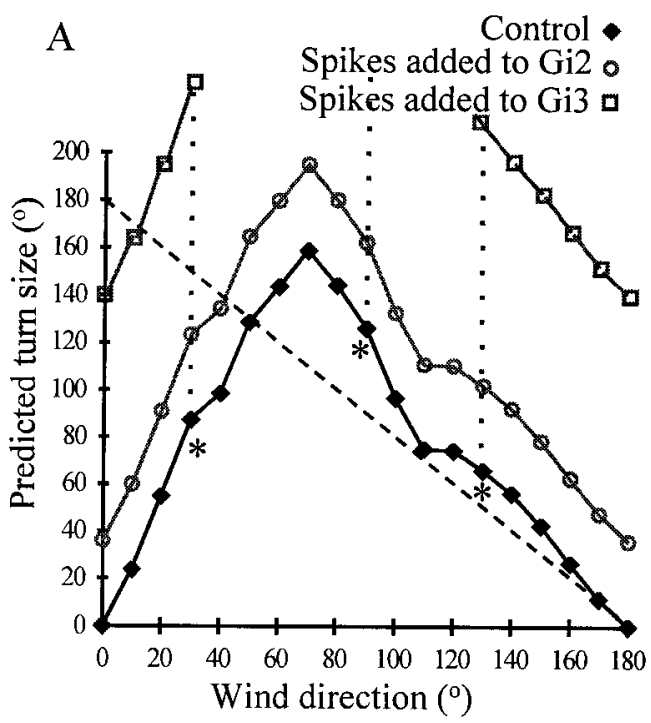

A

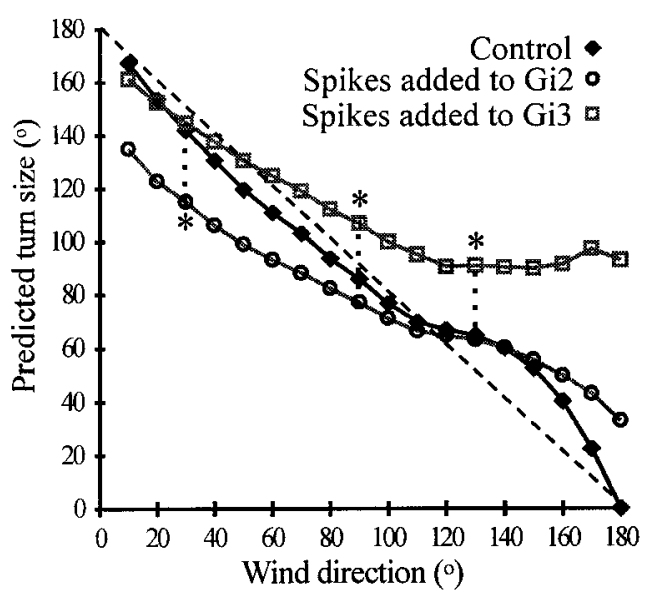

B

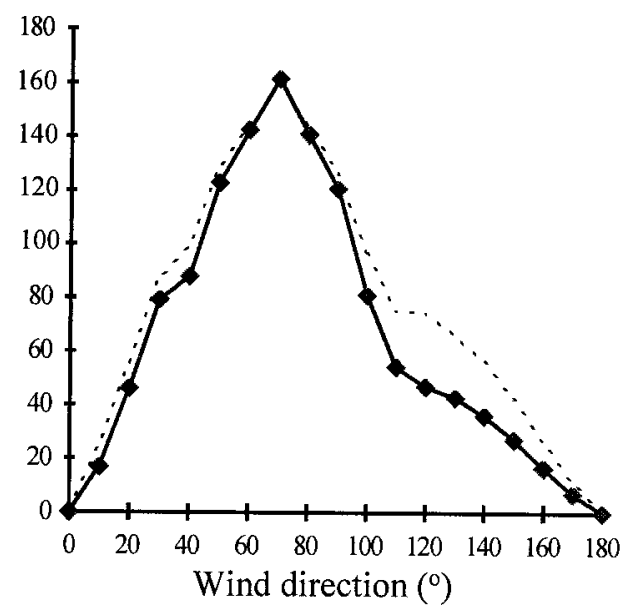

B

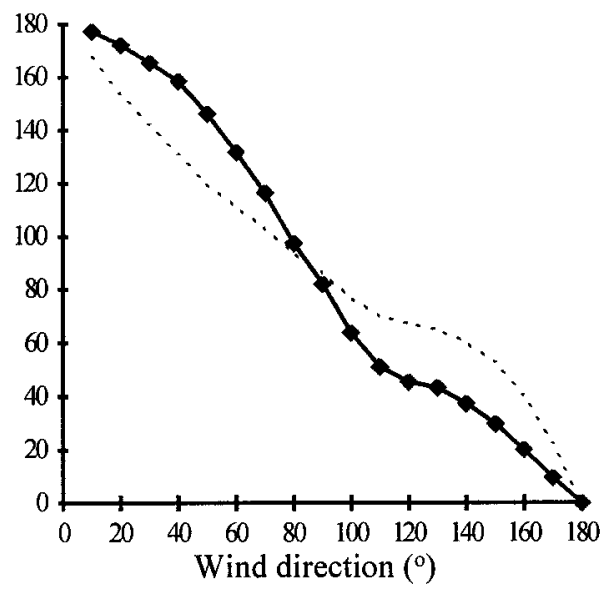

Figure 2. Sizes of left turns predicted by the steering wheel model in response to wind stimuli from different right angles, from near $0^{\circ}$ ("head-on" wind) to $180^{\circ}$ (wind from rear). $A$, Dashed line indicates the theoretical turn sizes made by a cockroach that would turn perfectly away from the wind source. Filled diamonds, Simulation involving all three left and three right GIs. Open symbols, Turn sizes predicted by the model after adding five spikes to either right GI 2 or GI3. Asterisks, Specific predictions tested in the physiological experiments. (The effect of adding spikes to GI3 is greater than GI2, because GI3 has a higher weighting; see Results for explanation.) $B$, Dashed line, The same simulation as shown by the filled diamonds in $A$. The filled diamonds here show the predicted turn size when left and right GIs 1 are deleted from the simulation.

Figure 3. Sizes of left turns predicted by the population vector model in response to wind stimuli from different right angles, as in Figure 2. A, Dashed line indicates perfect turning exactly away from the wind source. Filled diamonds, Simulation involving all three left and three right GIs. Open symbols, Turn sizes predicted by the model after adding five spikes to either right GI2 or GI3. Asterisks, Specific predictions tested in the physiological experiments. $B$, Dashed line, The same simulation as shown by filled diamonds in part $A$. The filled diamonds here show the predicted turn size when left and right GIs 1 are deleted from the simulation.

over $2 / 3$ of the range of wind directions, however, the steering wheel is reasonably suitable as a candidate model. Interestingly, the model was very robust to the removal of the least directional of the GIs, namely left and right GI1, after which the prediction of the model was nearly unchanged (Fig. 2B). Without GI1, the RMS was 53.

We next simulated the escape behavior based on the population vector model. For this, we calculated the population vector as in Figure $1 C$, using all six GIs, for right wind directions between $0^{\circ}$ and $180^{\circ}$. (We used $90^{\circ}$ as the preferred stimulus direction for GI1.) Finally, we reversed the direction by $180^{\circ}$ to obtain the turn angle, again on the assumption of perfect turning away from the wind source.

Figure $3 A$ (solid diamonds) shows the turn size as a function of wind direction. The dashed line represents perfect turns, exactly away from the wind direction. The model gives a very close fit to these perfect turns (RMS of $1.86^{\circ}$ ). The maximal divergence between the estimated turn and the perfect turn is $20^{\circ}$. It should be noted that, with this model, we did not perform any kind of optimization on the weightings of the different vectors of the GIs. It is likely that such optimization would have improved the performance (Salinas and Abbott, 1994). Nevertheless, our calculations demonstrate that even a nonoptimized population vector model can account well for the cockroach's escape behavior. As with the steering wheel model, the results were little changed when we removed GI1 from the calculation: RMS of 1.83 (Fig. 3B).

Both the steering wheel and the population vector models can account for the results of a recent behavioral-physiological experiment (Levi and Camhi, 2000) in which spikes were added to the right GI 3 during wind stimulation. With wind from either $90^{\circ}$ or $130^{\circ}$ right, the addition of these spikes increased turn size. Figure 4, $A$ and $B$, shows that this is expected according to both the steering wheel (left panels) and population vector (right panels) models. Specifically, for the steering wheel model, adding spikes to the right GI3 lengthens the curved arrow RGI3 (i.e., adds a large counterclockwise component) and therefore leads to a larger left turn than in $A$ above. As for the population vector model, adding spikes to the right GI3 leads to a calculation of the wind angle as closer to the right front and therefore leads to a larger left turn than in $A$ above. This is shown more systematically in Figures $2 A$ and $3 A$ (open squares).

More significantly, the two models give opposite predictions for the addition of spikes to right GI2 during the delivery of wind stimuli from $90^{\circ}$ right. The steering wheel model predicts an enlarged turn, whereas the population vector model predicts a smaller turn relative to controls. As Figure $4 C$ (left panel) shows 

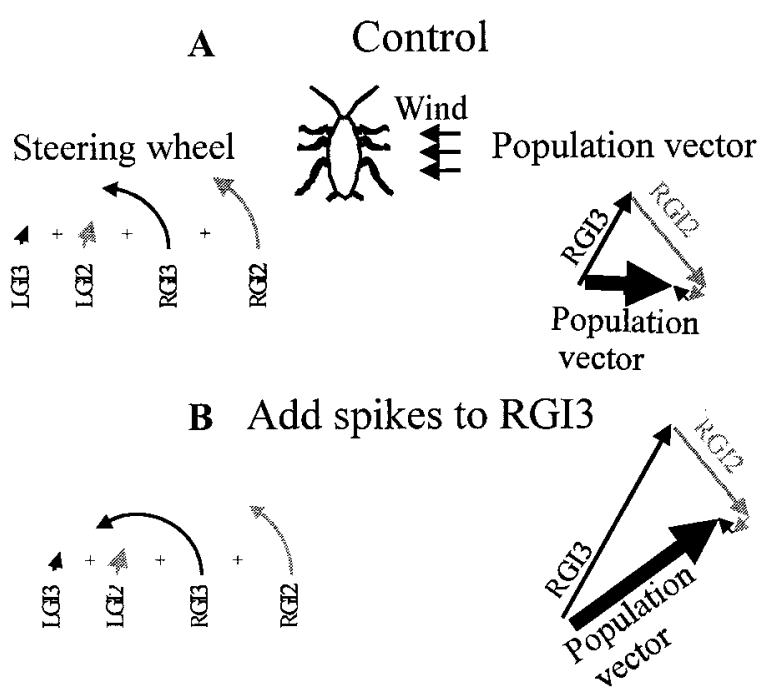

C Add spikes to RGI2

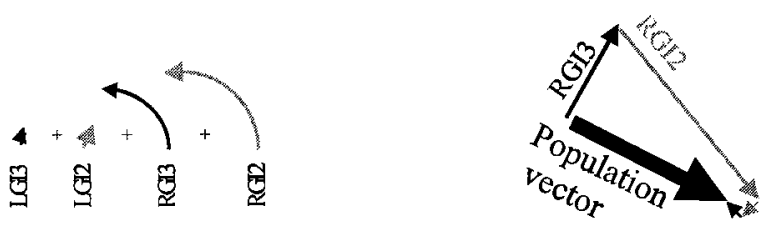

Figure 4. Calculations of the effects via the steering wheel and the population vector codes of adding five spikes to right GIs 3 versus right GI2. A, Control, With no spikes added. Calculations shown are from Figure $1 B$, bottom panels. $B$, The arrow for right GI3 is lengthened, indicting addition of spikes. See Results for explanation. $C$, The same for right GI2. See Results for explanation.

for the steering wheel model, adding spikes to right GI2 lengthens the curved arrow RGI2 (i.e., adds a large counterclockwise component) and thus leads to a larger left turn than in $A$ above. In contrast, as Figure $4 C$ (right panel) shows for the population vector model, adding spikes to right GI2 leads to a calculation of the wind angle as closer to the right rear than in $A$ above and thus to a smaller left turn. This is shown explicitly for different wind angles in Figure $2 A$ and $3 A$ (open circles).

\section{Steering wheel versus population vector models: an experimental test}

To distinguish between the two models, we delivered wind puffs from either $30^{\circ}$ or $90^{\circ}$ right and attempted to increase the spike frequency of GI2 by injecting a train of electrical pulses of various frequencies. We chose $90^{\circ}$ right to parallel the earlier tests on GI3 at this same angle (Levi and Camhi, 2000) and $30^{\circ}$ right as an angle at which the population vector model predicts an especially large effect of adding spikes to GI2 (Figure $3 A$.)

However, we encountered a technical problem in attempting to add spikes to GI2 during wind stimulation. The response of this cell to wind begins with a remarkably high spike frequency, over 600 spikes/sec, more than either GIs 1 or 3 (Westin et al., 1977). For this reason, it was impossible to increase the spike frequency at the beginning of the burst, and in fact our attempts to do so generally decreased spike frequency, at least on the first two interspike intervals after the wind stimulus.

Therefore, we instead began by photoablating GI2, as described in the Materials and Methods. The effect of photoablation on turn direction should be opposite that of adding spikes to this

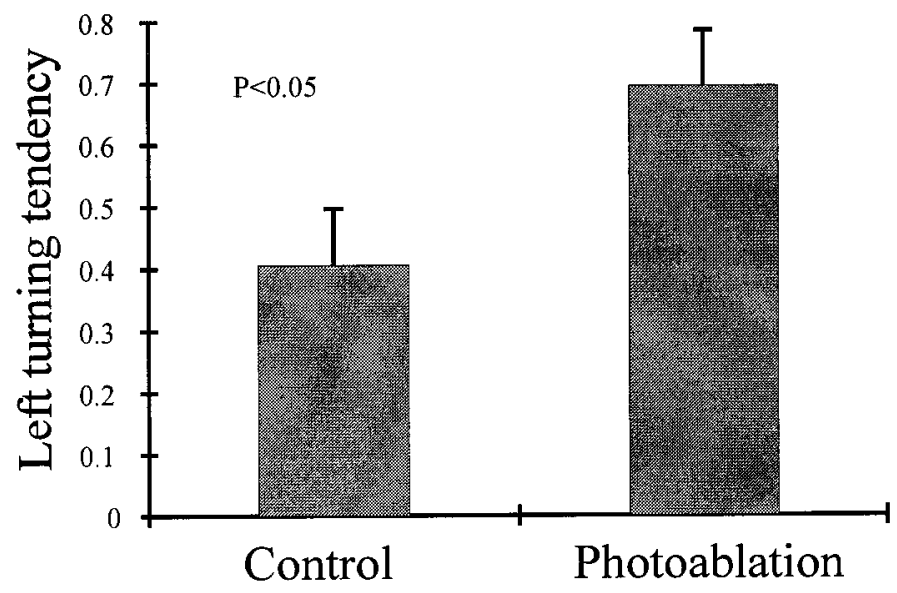

Figure 5. Effect of photoablation of right GI2 on the left-turning tendency. See Results for explanation.

cell. Thus, the population vector model would predict an increase of the left turn size, whereas the steering wheel model would predict a decrease. We delivered wind puffs, initially from $90^{\circ}$ right, to each of five animals, three times before and three times after the photoablation. The photoablation resulted in a significant increase of left-turning tendency ( $p<0.05$; Mann-Whitney test), consistent with the population vector model (Fig. 5).

In a separate group of 10 cockroaches, we followed the photoablation with subsequent addition of spikes to GI2. We first gave three wind puffs from $90^{\circ}$ right to each of these animals. We then performed the photoablation and gave three more puffs. Then, simultaneous with each of three more puffs, we delivered an electrical stimulus train to the more anterior region of the GI2 axon, as described in the Materials and Methods. These photoablation and electrical stimulation procedures are known not to effect the adjacent unfilled GIs (Libersat et al., 1989; Mizrahi and Libersat, 1997).

Figure $6 A$ (top trace) shows, from a trial on one of these 10 animals, the response to wind of right GI2 before photoablation. The middle trace shows the absence of spikes in response to wind after photoablation, and the bottom trace shows the response to a train of electrical stimulus pulses at $300 / \mathrm{sec}$. In this, as in each of the animals of this experiment, the time from the onset of the wind stimulus to the onset of the first spike differed by $<1 \mathrm{msec}$ in the top trace (control) versus the bottom trace (experimental). In the bottom trace, however, the electrically evoked spike train continued until well after the beginning of the escape behavior.

In the example shown, the photoablation alone caused the cockroach's left-turning tendency to increase by $20 \%$ (Fig. 6B). (This change is similar to that of Fig. 5.) Then, adding spikes to the GI returned the left-turning tendency to its prephotoablated level.

Figure $7 A$ shows the mean wind-evoked responses of all 10 GI2s from this experiment. It was this response that we eliminated completely by the photoablation. The dashed line shows the $300 \mathrm{~Hz}$ response to our electrical stimulation, well below the prephotoablation spike frequency of the cell in response to wind.

Of the 10 animals tested, two did not show any change in their left-turning tendency after photoablation. Therefore, we could not use them to test the restorative effect of GI2 activity. Figure $7 B$, therefore, shows the results of only the remaining eight animals. The effect of photoablation was a significant increase in turning tendency ( $p<0.05$; Wilcoxon paired test). There was 
A
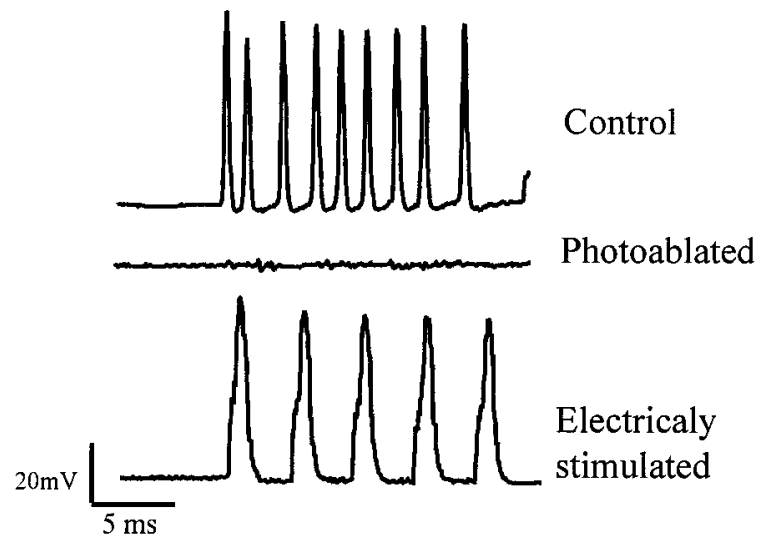

B

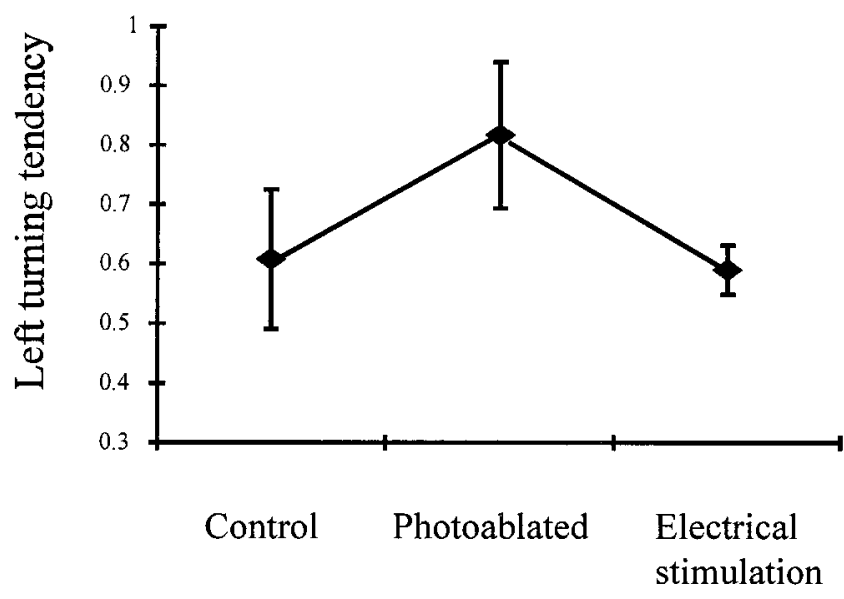

Figure 6. A sample experiment. $A$, Intracellular recordings from the right GI2 before (top trace) and after (middle trace) photoablation, and with subsequent electrical stimulation (bottom trace). $B$, The effect of photoablation and of subsequent electrical stimulation of GI2 in the same animal as in $A$. The graph includes three trails for each treatment (means \pm SEM).

then a significant reduction in left-turning tendency in response to the subsequent addition of spikes to GI2 $(p<0.05$; Wilcoxon paired test). This mean reduction equaled $60 \%$ of the increase in left-turning tendency that had occurred with the photoablation alone in these same eight animals.

These results of both the photoablation and the spike addition tests are consistent with the predictions of the population vector, and not the steering wheel, model (Fig. 4C). This, together with the far better performance of the population vector model (compare Figs. 2, 3) lend much stronger support to the population vector than the steering wheel model.

\section{DISCUSSION}

In the present paper, we have attempted to distinguish between two possible models to account for how neurons collaborate with one another to determine the direction of a particular behavior. Our models involved few assumptions about the properties of the neurons involved; for instance, they used only mean spike frequencies. And yet one of them (population vector) reconstructed the directional behavior quite accurately and the other (steering wheel) somewhat so.

To discriminate between the steering wheel and the population vector mechanisms, we manipulated the neural code experimentally by affecting a single GI and analyzed the behavioral effect of this on the strength of the cockroach's turning tendency away from the wind. Whereas results we had obtained previously using this method on GI3 were consistent with both the steering wheel and the population vector mechanisms, our tests on GI2 supported selectively the latter of these mechanisms.

The photoablation of right GI2, performed in two separate groups of cockroaches, gave rise to larger left-turning tendencies than in control trials before the photoablation. Because the photoablation tests on each animal had to be performed after the control tests, it was possible that the effect on the behavior resulted from the timing of the trials and the consequent difference in the state of the animal. However, the fact that photoablation, which would occur when the preparation had somewhat deteriorated, actually enhanced the response argues against this. And indeed, the reduction of the left-turning tendency that resulted from the subsequent addition of spikes more anteriorly in the photoablated GI2 axon likewise is consistent with the direction of effect caused by the photoablation.

An additional prediction of a population vector model is that increasing the number of spikes in a given GI should draw the cockroach's perception of wind direction from either side toward the preferred direction of this GI. For instance, as shown on the right side of Figure 4, $A$ and $B$, adding spikes to the right GI3 draws the perception of the direction of the wind from $90^{\circ}$ right (the actual wind angle) to a more anterior direction. Figure 8 shows what one would expect if the wind had come, instead, from the opposite side of the preferred direction of GI3: specifically, from $90^{\circ}$ left. Without adding any spikes to GI3, the population vector would point to the actual wind direction, $90^{\circ}$ left, so the turn would be to the right. Now, if one were to add sufficient spikes to the right GI3, the population vector would point not toward the cockroach's left side, but rather high to the right. Thus, the direction of the turn would be reversed (toward the right), and in fact the turn would be a large one because the population vector points specifically toward the front on the right side. Such a result would indicate that the right GI3 can draw the perception of wind direction toward its preferred direction from either side. In fact, the result of Figure 8, a large, reversed turn, is just what is obtained when the GI3 opposite the wind stimulus is stimulated with a sufficiently high-spike frequency (Liebenthal et al., 1994; Levi and Camhi, 2000). In contrast, the steering wheel model would predict a small turn, because large positive and negative numbers would be added together, resulting in a small sum (Fig. 1B, bottom panel). This further supports the population vector model of the cockroach.

An additional test of the population vector model in this system, at least in principle, would be to deliver wind from the preferred direction of a GI and then add spikes to this GI. This should not affect the cockroach's turning tendency. This can be seen in Figure $3 A$ in which adding spikes to right GI 2 with the wind from $120^{\circ}$, or to right GI3 with the wind from $30^{\circ}$, does not affect the left-turning tendency. These experiments would be nearly impossible to perform, however, because they would require adding spikes to the already maximal wind response of the given GI. In principle, one could overcome this problem by greatly lowering the wind intensity and thus the wind-evoked spike frequency. Then it should be possible to increase the spike frequency by intracellular electrical stimulation. However, in these dissected preparations, strong wind stimuli are required to obtain any behavioral response. 
A

Figure 7. Summed data from photoablation and subsequent electrical stimulation of right GI2. $A$, Mean instantaneous spike frequency of GI2 in response to wind puffs from $90^{\circ}$ (control). The dashed line represents the frequency in response to electrical stimulation after photoablation. $B$, The left-turning tendency of the pooled animals before and after photoablation, and after electrical stimulation, of GI2. See Results for explanation.

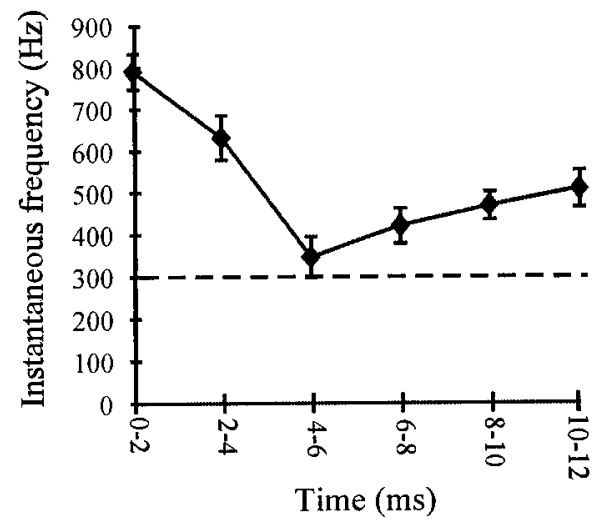

A

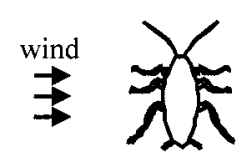

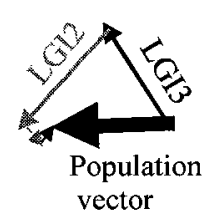

B

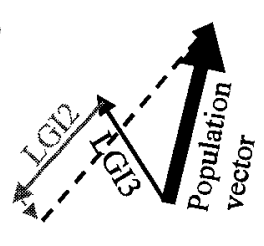

Figure 8. Population vector model for wind from $90^{\circ}$ left. $A$, Intact situation. $B$, Addition of spikes to right GI3 changes the population vector from $90^{\circ}$ left to a right frontal angle. This would produce a large left turn.

An additional conclusion that can be drawn from the experiments presented here concerns fine temporal patterning. It has been suggested (Abeles and Gerstein, 1988; Theunissen and Miller, 1991; Theunissen and Miller, 1995; Engel et al., 1997), and in some cases shown (Gelperin et al., 1993; Laurent et al., 1996), that fine temporal patterning serves as a neural code and thus can, in principle, affect behavior. In previous experiments on the cockroach GIs and escape behavior, it has been found that adding electrically evoked spike trains to a given GI produces consistent changes in the turn direction (Liebenthal et al., 1994; Levi and Camhi, 2000). This suggested that fine temporal patterning was not a major factor, if indeed it is involved at all, in the cockroach GI code for direction. However, an objection to this interpretation had always been that the interactions of the wind stimulation and electrical stimulation produced a spike train with some jitter in the timing of particular spikes. This jitter could conceivably play some role in specifying wind direction.

In the present experiments, however, as we first photoablated the GI and then stimulated its axon electrically more anteriorly, the firing pattern of this GI in the experimental trials was a pure frequency (Fig. 6A, third trace). Yet, even without any frequency jitter, this electrical stimulation very substantially influenced the animal's turning tendency in the expected direction (Figs. 6B, $7 B$ ). It seems clear, then, that the cockroach GI system works primarily on the basis of the number or frequency of spikes and not on fine temporal parameters.

The population vector diagrams of Figures 1, 4, and 8 are intended to show the kind of computation the cockroach's nervous system might perform in determining the wind direction (and hence, the appropriate turn direction). Within the nervous system, however, these computations are based on the synaptic interactions among particular neurons. Both GIs 2 and 3 are known to activate the thoracic interneurons (TIs) that constitute the next step in the neural process (Ritzmann and Pollack, 1986, 909-924.
B

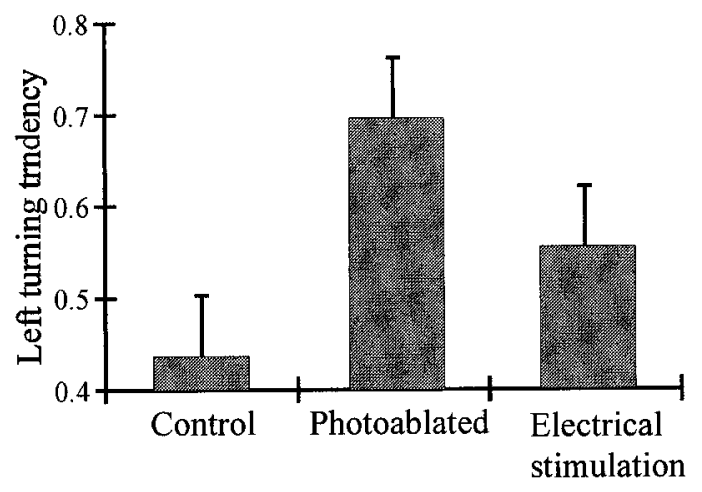

1988, 1990). The TIs receive only excitatory inputs from the GIs. Spatial and temporal summation are essential for spike generation in these cells, and further summation is needed from the TIs to evoke a motor response.

The population vector model suggests at least partial segregation of input from GI2 versus GI3 to different groups of TIs, because each of these GIs promotes a turns of a different size. Indeed, the TIs themselves are directionally tuned, although less sharply than GIs 2 or 3, suggesting some segregation of their inputs. Moreover, a TI that is not connected to GI3 is found to be generally tuned primarily to rear winds, as would be predicted by the frontal receptive field of GI3 (Ritzmann and Pollack, 1988).

The patterns of GI and TI connectivity are not yet sufficiently understood to translate the population vector code suggested by the present study into specific sets of neural connections. This should be possible, however, and indeed a key advantage of studying population vector coding in a simple system is the prospect of revealing its cellular basis.

It seems remarkable that the population vector mechanism, first demonstrated in the monkey brain, now appears also to apply to an insect nervous system. Moreover, recent results suggest that it also applies to the tactually evoked bending response of the leech (Lewis and Kristan, 1998) at the level of sensory cells. Although the cellular properties of individual neurons are known to have been preserved since the evolution of very early living forms, much less is known about the evolutionary conservation of multineuronal systems of integration. Whether population vector coding is such an example or is a case of parallel evolution remains to be determined.

\section{REFERENCES}

Abeles M, Gerstein G (1988) Detecting spatiotemporal firing patterns among simultaneously recorded single neuron. J Neurophysiol 60 :

Camhi JM (1988) Escape behavior in the cockroach: distributed neural processing. Experientia 44:401-408.

Camhi JM, Levy A (1988) Organization of a complex movement: fixed and variable components of the cockroach escape behavior. J Comp Physiol [A] 163:317-328.

Camhi JM, Tom W (1978) The escape behavior of the cockroach Periplaneta americana. I. Turning response to wind puffs. J Comp Physiol [A] 128:193-201.

Comer C (1985) Analyzing cockroach escape behavior with lesions of individual giant interneurons. Brain Res 335:342-346.

Dowd J, Comer C (1988) The neural basis of orienting behavior: a computational approach to the escape turn of the cockroach. Biol Cybern 60:37-48.

Eaton RC, DiDomenico R, Nissanov J (1991) Role of the Mauthner cell 
in sensorimotor integration by the brain stem escape network. Brain Behav Evol 37:272-285.

Engel AK, Roelfsema PR, Fries P, Brecht M, Singer W (1997) Role of the temporal domain for response selection and perceptual binding. Cereb Cortex 7:571-582.

Evoy WH, Kennedy D (1967) The central nervous organization underlying control of antagonistic muscles in the crayfish. I. Types of command fibers. J Exp Zool 165:223-238.

Gelperin A, Rhines LD, Flores J, Tank DW (1993) Coherent network oscillations by olfactory interneurons: modulation by endogenous amines. J Neurophysiol 69:1930-1939.

Georgopoulos AP, Schwartz AB, Kettner RE (1986) Neuronal population coding of movement direction. Science 233:1416-1419.

Groh JM, Born RT, Newsome WT (1997) How is a sensory map read out? Effects of microstimulation in visual area MT on saccades and smooth pursuit eye movements. J Neurosci 17:4312-4330.

Kennedy D, Evoy WH, Dane B, Hanawalt JT (1967) The central nervous organization underlying control of antagonistic muscles in the crayfish. II Coding of position by command fibers. J Exp Zool 165:239-248.

Kolton L, Camhi JM (1995) Cartesian representation of stimulus direction: parallel processing by two sets of giant interneurons in the cockroach. J Comp Physiol [A] 176:691-702.

Laurent G, Wehr M, Davidowitz H (1996) Temporal representations of odors in an olfactory network. J Neurosci 16:3837-3847.

Levi R, Camhi J (2000) Wind direction coding in the cockroach escape response: winner does not take it all. J Neurosci, 20:3814-3821.

Lewis JE, Kristan Jr WB (1998) A neuronal network for computing population vectors in the leech. Nature 391:76-79.

Libersat F, Mizrahi A (1996) In situ visualization and photoablation of individual neurons using a low cost fiber optic based system. J Neurosci Methods 67:157-162.

Libersat F, Selverston A, Camhi JM, Goldstein RS (1989) Photoinactivation of a portion of a neuron for long term studies of its role in behavior. J Exp Biol 142:453-459.

Liebenthal E, Uhlmann O, Camhi JM (1994) Critical parameters of the spike trains in a cell assembly: coding of turn direction by the giant interneurons of the cockroach. J Comp Physiol [A] 174:281-296.

Miller JP, Selverston AI (1979) Rapid killing of single neurons by irradiation of intracellularly injected dye. Science 206:702-704.

Mizrahi A, Libersat F (1997) Independent coding of wind direction in cockroach giant interneurons. J Neurophysiol 78:2655-2661.

Nye SW, Ritzmann RE (1992) Motion analysis of leg joints associated with escape turns of the cockroach, Periplaneta americana. J Comp Physiol [A] 171:183-194.

Ritzmann RE, Pollack AJ (1986) Identification of thoracic interneurons that mediate giant interneuron-to-motor pathways in the cockroach. J Comp Physiol [A] 159:639-654.

Ritzmann RE, Pollack AJ (1988) Wind-activated thoracic interneurons of the cockroach. II. Patterns of connection from ventral giant interneurons. J Neurobiol 19:589-611.

Ritzmann RE, Pollack AJ (1990) Parallel motor pathways from thoracic interneurons of the ventral giant interneuron system of the cockroach, Periplaneta americana. J Neurobiol 21:1219-1235.

Salinas E, Abbott LF (1994) Vector reconstruction from firing rates. J Comput Neurosci 1:89-107.

Salzman CD, Newsome WT (1994) Neural mechanisms for forming a perceptual decision. Science 264:231-237.

Sparks DL, Holland R, Guthrie BL (1976) Size and distribution of movement fields in the monkey superior colliculus. Brain Res 113:21-34.

Theunissen F, Miller J (1995) Temporal encoding in nervous systems: a rigorous definition. J Comput Neurosci 2:149-162.

Theunissen FE, Miller JP (1991) Representation of sensory information in the cricket cercal sensory system. II. Information theoretic calculation of system accuracy and optimal tuning-curve widths of four primary interneurons. J Neurophysiol 66:1690-1703.

Westin J, Langberg JJ, Camhi JM (1977) Responses of giant interneurons of the cockroach Periplaneta americana to wind puffs of different directions and velocities. J Comp Physiol [A] 121:307-324.

Wine J, Krasne F (1972) The organization of the escape behavior in the crayfish. J Exp Biol 56:1-18. 